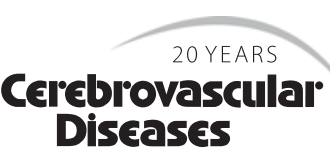

Diseases

\title{
A Simultaneous EEG and Transcranial Doppler Technique to Investigate the Neurovascular Coupling in the Human Visual Cortex
}

\author{
B. Rosengarten M. Kaps \\ Department of Neurology, Justus-Liebig University of Giessen, Giessen, Germany
}

\section{Key Words}

Doppler ultrasound $\cdot$ Visual evoked potential $\cdot$ Cortical activation $\cdot$ Neurovascular coupling $\cdot$ Cerebral blood flow

\begin{abstract}
Due to lacking energy stores, high energy consumption and dependency on a strict aerobic glycolysis, the brain is very dependent on an adequate blood supply of active neurons. Adequate regulation of the functionally induced local cerebral blood flow is assured by neurovascular coupling. Our understanding of its role in different disease processes has improved considerably in the last few years. Consequently, a diagnostic tool is needed which allows an easy and costeffective investigation of the coupling in clinical conditions. A combined EEG and Doppler technique might be suitable in this regard. This review gives a short overview of the neurophysiological background and the techniques, before validation studies and first clinical applications are addressed.
\end{abstract}

Copyright $\odot 2009$ S. Karger AG, Base

\section{Cerebral Circulation}

The brain is highly dependent on adequate perfusion because it has a high energy consumption of approximately $23 \mathrm{~J} / \mathrm{s}=23 \mathrm{~W}$, but lacks adequate stores of oxygen or energy substrate $[1,2]$. This explains why the brain, although only representing $2 \%$ of the body weight, re- ceives $15 \%$ of the cardiac output consuming $20 \%$ of the total body oxygen and $25 \%$ of total body glucose. The high energy needs can only be assured by aerobic glycolysis in which $1 \mathrm{~mol}$ glucose results in $38 \mathrm{~mol}$ adenosine triphosphate, whereas anaerobic glycolysis only produces 2 mol of the energy molecule [3]. From the stroke research of the last few years it is known that the brain becomes more sensitive to inappropriate perfusion with increased duration of impaired blood flow. While it can compensate even a strong decline in blood flow for a short time period, it reacts very sensitively to a chronic mismatch of adequate perfusion. A reduction of only $10 \%$ from the ideal perfusion leads to protein synthesis disturbance in neurons if it lasts for several hours or days [2]. Because of this dependency on the momentary blood flow, we can distinguish two effective, fast-regulated and fine-tuned vasoregulative mechanisms in the brain: cerebral autoregulation compensates for cerebral perfusion pressure changes, whereas neurovascular coupling adapts local cerebral blood flow in accordance with the underlying neuronal activity $[1,4]$. In what follows, we will concentrate on the neurovascular coupling mechanism.

\section{Neurovascular Coupling}

Historically, one of the first reports on the functional dependency of the cerebral blood flow in relation to the brain activity goes back to an Italian physician. In 1890,

\section{KARGER}

Fax +41613061234 E-Mail karger@karger.ch www.karger.com (c) 2009 S. Karger AG, Basel

$1015-9770 / 10 / 0293-0211 \$ 26.00 / 0$

Accessible online at:

www.karger.com/ced
Prof. Dr. med. B. Rosengarten

Justus-Liebig University of Giessen

Department of Neurology, Am Steg 14, DE-35392 Giessen (Germany)

Tel. +4964199 45301, Fax +496419945309

E-Mail bernhard.rosengarten@ neuro.med.uni-giessen.de 
Angelo Mosso [5] presented data from a patient with an open skull defect in whom he found a functionally related change in cerebral perfusion. Although the detailed mechanisms still remain to be elucidated, there are three main hypotheses how the coupling might be governed. One hypothesis assumes local interneurons or projection neurons from specialized brain stem nuclei (i.e. nucleus basalis of Meynert) to modulate the cerebral blood flow regulation since they have direct endings on arterioles [6-8]. Alternatively, it was shown that activity-related changes in local mediators can influence the vascular tone: changes in the extracellular ion content (potassium and hydrogen ions), depletion products of energy molecules (adenosine) or nitric oxide can mediate the vascular tone $[4,9]$. The most recent hypothesis assumes that the excitatory transmitter glutamate can be sensed by astrocytic receptors which then mediate the local vascular tone $[10,11]$.

\section{Transcranial Doppler Technique}

In the last few years, many studies have been conducted on the neurovascular coupling mechanism in experimental or clinical settings with different techniques. To assess the fast hemodynamic aspects of neurovascular coupling, many clinical studies have been performed with the transcranial Doppler technique [12]. Because of its limited spatial resolution, most investigations were done on the occipital cortex using visual stimuli insonating the P2 segment of the posterior cerebral artery. In the physiological range the hemodynamic response does not depend on variations of blood pressure, heart rate or orthostatic position [12-14]. The hydrogen ion concentration of the brain tissue has an important influence on neurovascular coupling. It can easily be varied by hyperor hypoventilation resulting in hypo- or hypercapnia. Hypercapnia increases and hypocapnia decreases the hydrogen ion content and therefore modulates the speed of vasoregulation: under hypercapnia the upstroke is declined and under hypocapnia improved. Furthermore, absolute flow velocity levels under hypercapnia increase whereas they decrease under hypocapnia. Interestingly, the relative changes in evoked flow velocities remain constant under the different blood gas conditions $[15,16]$. Since hyper- or hypocapnic state does not influence cortical activation, the findings assume a shift in the set point of neurovascular coupling. It has been shown that visual stimulation does not change the $\mathrm{pCO}_{2}$ concentration considerably so that routine $\mathrm{CO}_{2}$ measurements are not necessary [12]. If unspecific effects such as changes in blood pressure or ventilation are suspected, a parallel flow velocity recording can be performed in the middle cerebral artery, which usually shows no significant changes during visual stimulation.

The hemodynamic response depends on different features of the stimulus. It is positively correlated with an increased complexity degree, greater visual field section and is higher in chromatic than achromatic stimuli [12]. Using pattern reversal stimulation the responses also depend on contrast, luminance and flickering frequency of the stimulus $[17,18]$. The first significant hemodynamic responses appear approximately $700-1,000 \mathrm{~ms}$ after stimulation onset and are therefore a decade slower than the neuronal response [19]. The hemodynamic changes show a typical time course with a rapid initial increase, followed by an overshoot before stable flow velocity levels above the baseline level are reached [20]. The time until stabilization can vary between $15 \mathrm{~s}$ in simple to $30 \mathrm{~s}$ in complex stimuli. Although we did not find a habituation within the stimulation period of $40 \mathrm{~s}$ using complex visual stimulation tasks, some authors described a succeeding decline in the evoked flow velocity level with longer stimulation durations [21]. Besides habituation it has to be taken into consideration that simple stimulation paradigms can lead to a decline in attention and concentration. Moreover, they may result in a weaker visual fixation, which altogether leads to a secondary reduction in visual evoked potential (VEP) and coupled flow velocity response. Due to spontaneous fluctuations in cerebral hemodynamics and ventilation-related fluctuations (B waves), averaging of $8-10$ cycles is widely recommended increasing the signal-to-noise ratio. The Doppler technique measures blood flow velocity rather than blood flow. Therefore, most authors perform a transformation to relative data since these data correlate quite well with flow changes. This correlation exists because the basal cerebral vessels do not contribute to the neurovascular coupling and consequently remain constant in diameter $[20,22]$. The transformation of data also gains independence from the insonation angle. Setting the resting flow velocity level to zero the dynamics of the flow velocity regulation can be described in terms of a control system model $[23,24]$. The equation $\mathrm{G}(\mathrm{s})$ for the transfer function resembles a proportional-derivative second time delayed system and is given in the frequency dominant form as follows:

$$
G(s)=\frac{K \cdot\left(1+T_{V} \cdot s\right)}{\frac{s^{2}}{\omega^{2}}+2 \cdot \xi \cdot \frac{s}{\omega}+1}
$$




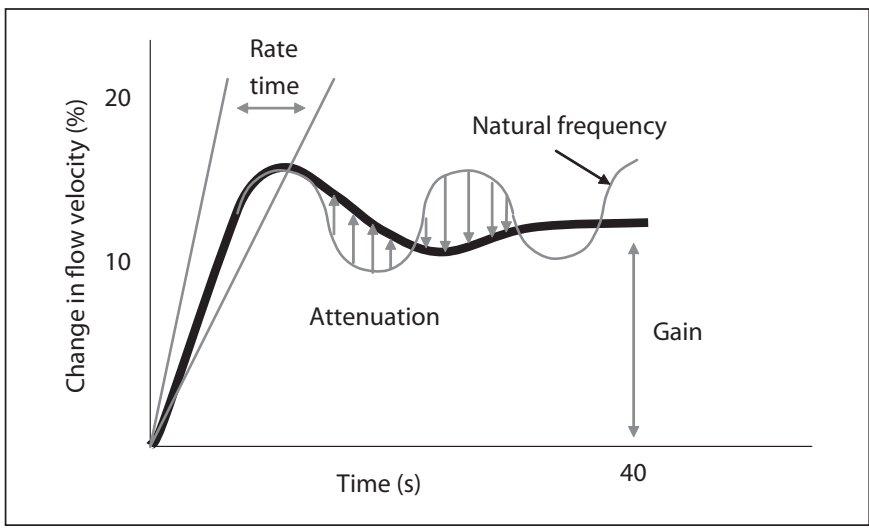

Fig. 1. The typical hemodynamic response in the posterior cerebral artery due to visual stimulation is shown. The flow velocity of the resting phase is set to zero. The evoked flow velocity responses are given in relative changes in relation to baseline. With onset of stimulation, flow velocities increase rapidly, overshoot and then stabilize on a constant level. The influence of the different control system parameters is illustrated. The gain parameter describes the difference to baseline at stable flow velocity conditions, whereas the rate time describes the speed of the initial upstroke. The parameters' natural frequency and attenuation describe the oscillating features of which attenuation describes the dampening, and natural frequency the ideal undampened oscillation of the system.

The parameters are gain, natural frequency, attenuation and rate time. Although abstract and mathematical, the parameters describe different characteristics of the hemodynamic response. The gain parameter $K$ specifies the flow velocity difference between the resting and stimulation phase under steady-state conditions. The rate time $\left(T_{v}\right)$ parameter specifies the steepness of the initial upstroke of the evoked flow velocity curve. This parameter characterizes the feed-forward properties of the system which improves the initial speed of the compensative regulatory process. The natural frequency and attenuation parameters describe oscillating features of the regulative system and represent, together with the gain parameter, the feedback characteristics of the neurovascular coupling. Whereas the natural frequency denotes oscillation as if the system were nondampened, the attenuation parameter indicates dampening in accordance with the elastic properties of the vascular system. The influences of the parameters on the evoked flow velocity responses are illustrated in figure 1 . Since the structures and mediator systems of neurovascular coupling are complex, the parameters are not specific for single physiological processes or distinct regulative steps of the cou-

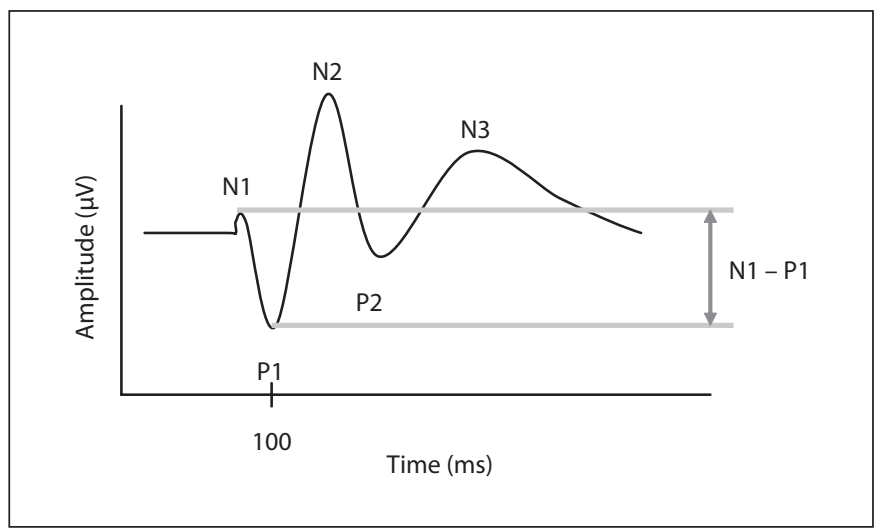

Fig. 2. The transient VEP with the typical peaks is shown. Usually the potential differences are specified. The early N1 - P1 amplitude difference is assumed to be generated from the primary visual cortex, whereas later amplitudes stem from higher cortical areas.

pling. However, there are hypotheses that the nitric oxide system is predominantly related to the rate time parameter, whereas the shifts in hydrogen ions or adenosine concentration can regulate the later phases of the coupling $[9,25]$. However, most cerebrovascular disease processes show a typical pattern with an early increase in the attenuation parameter (i.e. state of endothelial dysfunction) before a decline in the gain parameter as an indicator of a decreased vasoreactivity (i.e. beginning morphological changes) occurs $[26,27]$. The overshoot, which has often been used for evaluation, is not as appropriate since it depends on several control system parameters. Consequently, it consists of all their error terms which sum up to a considerably higher variability of the overshoot parameter. From a mathematical point of view, the use of the control system parameter is preferable to the overshoot parameter since it improves statistical comparisons and therefore sensitivity of the tests. Recently, the control system model has been validated in an animal model using somatosensory forepaw stimulation [24]. An advantage of using the Doppler technique is that it can easily be combined with EEG recording since signals do not interfere.

\section{EEG Technique}

EEG has long been used for determining VEP. In most cases, contrast-based pattern reversal stimulation is performed. Using lower pattern reversal frequencies, the 
typical so-called transient VEP waveform occurs, whereas higher reversal frequencies progressively lead to a sinusoidal steady-state VEP configuration. The transition occurs at around 4-7 Hz. The transient VEP is usually evaluated by determining the typical peaks which are named after a positive (negative values) or negative (positive values) deflection as shown in figure 2 [28]. VEP data are usually given as amplitude differences of typical peaks (i.e. N1 - P1 or N2 - P1 difference). The early amplitude differences (N1 - P1) are assumed to represent the activity of the primary visual cortical areas whereas later components indicate activity of higher visual cortical areas $[29,30]$. Since EEG allows continuous monitoring of cortical activity it might be possible to apply frequency analysis techniques to correlate between cortical activity and the Doppler signal.

\section{Combined EEG-Doppler Approach}

An advantage of the combined EEG-Doppler approach is that both techniques do not interfere with each other. This allows the use of commercially available instruments without further cost factors. We use a flexible Doppler headband or helmet in which the EEG electrodes are mounted on the defined positions in the fixation system of the device allowing a fast and simple application to the patient. The Doppler probes are mounted as described by the manufacturer. Basic research studies were performed with contrast-based visual stimuli and in most cases checkerboard-like stimulation paradigms were used. From these studies a close correlation between VEP amplitudes and evoked flow velocity responses (gain parameter) was found. A positive correlation was found for the contrast level, complexity degree or visual field section of the stimulation pattern, as can be seen in figure 3 $[17,18]$. Comparing initial versus later VEP amplitude differences a higher correlation of the initial amplitude differences with the gain parameter was found, whereas later components showed a succeeding decreasing correlation. This finding matches well with fMRI investigations which demonstrate high flow velocity changes predominantly in the primary visual cortex [21, 31]. Regarding flicker frequency modulation from 1 to 1.5 and $2 \mathrm{~Hz}$, no significant effects on either VEP or evoked flow velocity responses were found [17]. Taken together, the combined EEG- functional transcranial Doppler approach underlines the tightness of the coupling even in humans.

The lacking effect of the stimulation parameters on the other hemodynamic parameters indicates that these parameters mainly characterize vascular properties of the regulative system. Consequently they were found to be affected in different vascular disease processes [26, 27]. Regarding age-related effects on the coupling it can be followed from the literature that the coupling is comparable in healthy volunteers between 10 and 60 years of age and that it declines in elderly above 60 years of age [32-34]. Changes in other biological parameters in the higher aged have been published many times but it is still a matter of debate if the changes appear physiologically or indicate a pathological process.

Clinically, the combined EEG-Doppler approach has been used to differentiate between different types of dementia syndromes. Patients with a vascular type of dementia show a strong decrease to nearly absence of functionally activated hemodynamic responses, whereas a decline in visual amplitudes is less pronounced. This constellation supports the hypothesis of a primary uncoupling of the neurovascular coupling before a decline in potentials follows. Alzheimer patients without any vascular lesions on a cerebral MRI scan showed an increased attenuation parameter which normalized dosedependently under acetylcholine esterase inhibitor treatment. In animal studies, it was shown that the nucleus basalis of Meynert has cholinergic endings on the cerebral vessels and that a certain cholinergic tonus is necessary to stabilize the endothelial nitric oxide synthase [8]. Unselected Alzheimer patients presented more cerebrovascular lesions on the MRI scan and had a higher stroke risk as compared to non-Alzheimer controls $[35,36]$. Besides the change in the attenuation parameter, we found a smaller gain parameter, which was indicative of a decreased cerebrovascular reserve. The parameter was not influenced by an anticholinergic treatment and is assumed to be caused by an increased amyloid depletion in these patients. Medical effects on the neurovascular coupling or the differentiation of different headache subtypes were also investigated with the current approach.

\section{Outlook}

Taken together it appears that the combined EEG and Doppler technique is a valuable and sensitive tool to examine neurovascular coupling in clinical conditions. Using portable devices the examination can be performed at bedside and is well tolerated by the test persons. The technique is time and cost effective since it can be performed by a medical technician and within half an hour. 
Fig. 3. Demonstration of the dependence of the VEP amplitude (N1 - P1) and evoked flow velocity response (gain parameter) on different features of the visual stimulus. In the upper row (a), the dependence on the complexity degree of the stimulus is shown. With increasing complexity (decreasing check size), the evoked potentials as well as the coupled hemodynamic response increase. A similar relationship exists when increasing the size of the visual field (b). The changes between electrical and hemodynamic responses appear in a near-linear fashion (regression line between data pairs). Results are given as means and standard deviations from 9 healthy young volunteers.

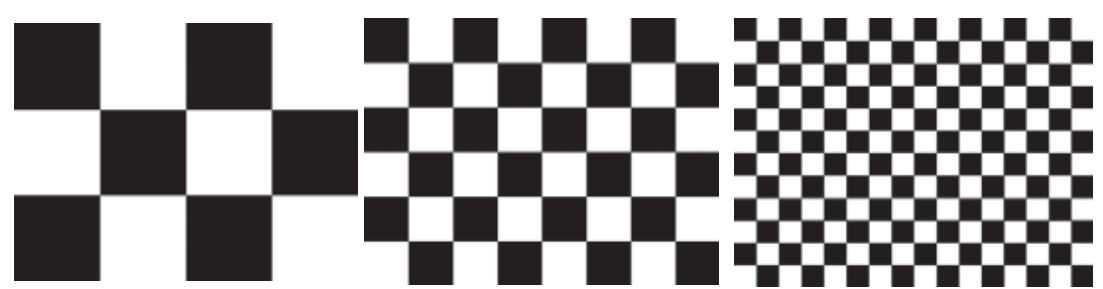

a
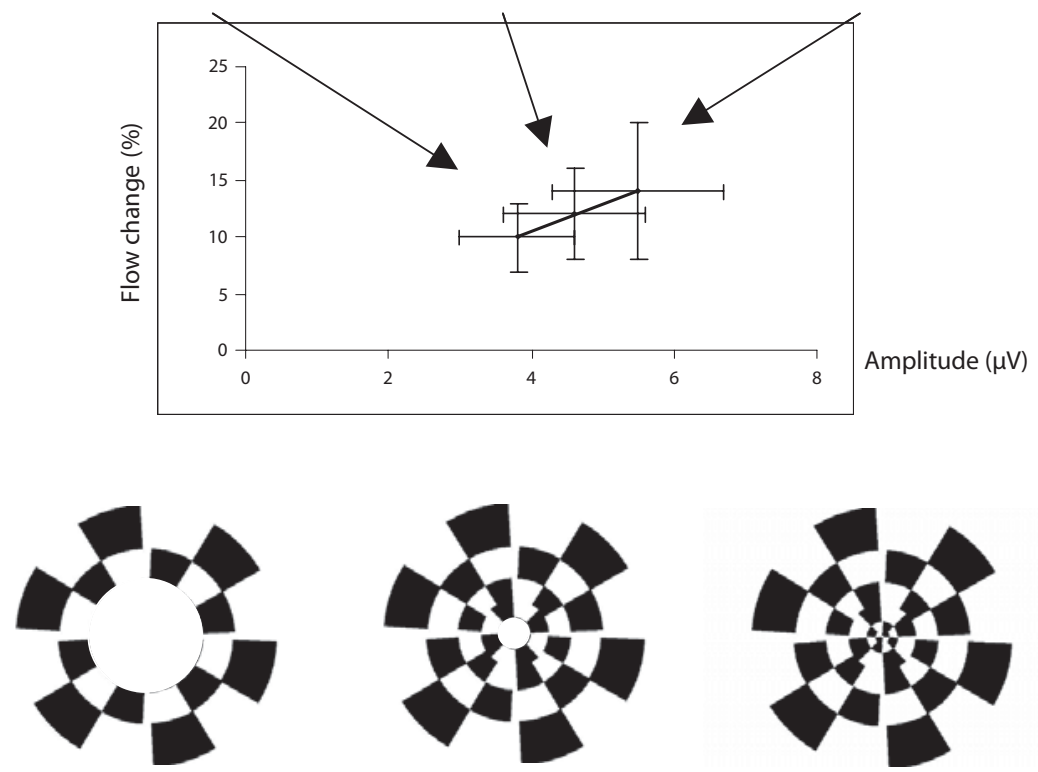

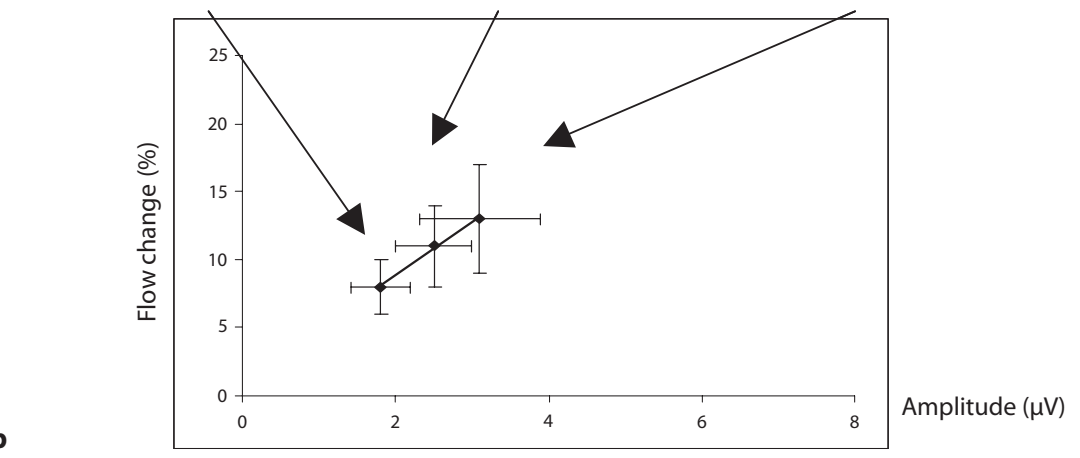

Amplitude $(\mu \mathrm{V})$

b
Limitations of the technique might result from noncorrectable vision deficits or an inappropriate temporal bone window. In our hands, and with newer Doppler probes, an insufficient temporal bone window in elderly is only a problem in about $5 \%$ of cases. Patients with epilepsy might be investigated with caution and the EEG traces should be studied by a skilled person online. The clinical application of the technique is manifold. It might aid in the differentiation between vascular, neurodegenerative or mixed dementia processes due to typical changes in the evoked potential and evoked flow patterns of the different diseases. Furthermore, the cascade of atherosclerotic disease processes, which generally start with an increased attenuation followed by a progressive decline in the gain parameter, might be studied before changes in evoked potential amplitudes occur. It might also aid in assessing efficacy of therapeutic interventions or determining medical effects on neurovascular coupling. 


\section{References}

1 Iadecola C: Neurovascular regulation in the normal brain and in Alzheimer's disease. Nature 2004;5:347-360.

$\checkmark 2$ Hossmann KA: Viability thresholds and the penumbra of focal ischemia. Ann Neurol 1994;36:557-565.

$\checkmark 3$ Sokoloff L: Energetics of functional activation in neural tissues. Neurochem Res 1999; 24:321-324

-4 Dirnagl U, Niwa K, Lindauer U, Villringer A: Coupling of cerebral blood flow to neuronal activation: role of adenosine and nitric oxide. Am J Physiol 1994;267:H296-H301.

5 Mosso A: Sulla circolazione del cervello dell'uomo. Atti R Accad Lincei 1880;5:237358.

6 Branston NM: Neurogenic control of the cerebral circulation. Cerebrovasc Brain Metab Rev 1995;7:338-349.

$\checkmark 7$ Iadecola C: Regulation of the cerebral microcirculation during neural activity - Is nitricoxide the missing link. Trends Neurosci 1993;16:206-214.

-8 Tong XK, Hamel E: Regional cholinergic denervation of cortical microvessels and nitric oxide synthase-containing neurons in Alzheimer disease. Neuroscience 1999;92:163175.

$\checkmark 9$ Kuschinsky W: Coupling of function, metabolism, and blood flow in the brain. Neurosurg Rev 1991;14:163-168.

10 Anderson CM, Nedergaard M: Astrocytemediated control of cerebral microcirculation. Trends Neurosci 2003;26:340-344.

- 11 Zonta M, Angulo MC, Gobbo S, Rosengarten B, Hossmann KA, Pozzan T, Carmignoto G: Neuron-to-astrocyte signaling is central to the dynamic control of brain microcirculation. Nat Neurosci 2003;6:43-50.

$\checkmark 12$ Conrad B, Klingelhöfer J: Dynamics of regional cerebral blood flow for various visua stimuli. Exp Brain Res 1989;77:437-441.

-13 Azevedo E, Rosengarten B, Santos R, Freitas J, Kaps M: Interplay of cerebral autoregulation and neurovascular coupling evaluated by functional TCD in different orthostatic conditions. J Neurol 2007;254:236-241.

-14 Rosengarten B, Budden C, Osthaus S, Kaps $\mathrm{M}$ : Effect of heart rate on regulative features of the cortical activity-flow coupling. Cerebrovasc Dis 2003; 16:47-52.
15 Ances BM, Greenberg JH, Detre JA: The effects of graded hypercapnia on the activation flow coupling response due to forepaw stimulation in alpha-chloralose anesthetized rats. Brain Res 2001;304:57-60.

16 Schmitz B, Böttiger BW, Hossmann KA Brief hypercapnia enhances somatosensory activation of blood flow in rat. J Cereb Blood Flow Metab 1996;16:1307-1311.

17 Rosengarten B, Molnar S, Trautmann J, Kaps M: Simultaneous VEP and transcranial Doppler ultrasound recordings to investigate activation-flow coupling in humans. Ultrasound Med Biol 2006;32:1171-1180.

18 Zaletel M, Strucl M, Rodi Z, Zvan B: The relationship between visually evoked cerebral blood flow velocity responses and visualevoked potentials. Neuroimage 2004;22: 1784-1789.

19 Klingelhöfer J, Wittich I, Sander D, Conrad B: Latencies of visually evoked perfusion changes in the posterior cerebral artery territory. J Neurol 1992;239:23-26.

20 Aaslid R: Visually evoked dynamic blood flow response of the human cerebral circulation. Stroke 1987; 18:771-775.

21 Obrig H, Israel H, Kohl-Bareis M, Uludag K Wenzel R, Muller B, Arnold G, Villringer A Habituation of the visually evoked potential and its vascular response: implications for neurovascular coupling in the healthy adult. Neuroimage 2002;17:1-18.

22 Kontos HA: Validity of cerebral arterial blood flow calculations from velocity measurements. Stroke 1989;20:1-3.

23 Rosengarten B, Huwendiek O, Kaps M: Neurovascular coupling and cerebral autoregulation can be described in terms of a control system. Ultrasound Med Biol 2001;27:189193.

24 Rosengarten B, Lutz H, Hossmann K-A: A control system approach for evaluating somatosensory activation by laser-Doppler flowmetry in the rat cortex. J Neurosci Methods 2003;130:75-81.

25 Buerk DG, Ances BM, Greenberg JH, Detre JA: Temporal dynamics of brain tissue nitric oxide during functional forepaw stimulation in rats. Neuroimage 2003;18:1-9.

-26 Rosengarten B, Dost A, Kaufmann A, Gortner L, Kaps M: Impaired cerebrovascular reactivity in type-1 diabetic children. Diabetes Care 2002;25:408-410.
27 Rosengarten B, Paulsen S, Molnar S, Kaschel R, Gallhofer B, Kaps M: Activation-flow coupling differentiates between vascular and Alzheimer type of dementia. J Neurol Sci 2007;257:149-154.

28 Bach M, Ullrich D: Contrast dependency of motion-onset and pattern-reversal VEPs: interaction of stimulus type, recording site and response component. Vision Res 1997;37: 1845-1849.

29 Baseler HA, Sutler EE, Klein SA, Carney T: The topography of visual evoked response properties across the visual field. Electroencephalogr Clin Neurophysiol 1994;90:6581.

30 Horton JC, Hoyt WF: The representation of the visual field in human striate cortex. Arch Ophthalmol 1991;109:816-824.

-31 Guy CN, Ffytche DH, Brovelli A, Chumillas J: fMRI and EEG responses to periodic visual stimulation. Neuroimage 1990;10:125148 .

32 Rosengarten B, Aldinger C, Spiller A, Kaps M: Neurovascular coupling remains unaffected during normal aging. J Neuroimaging 2003;13:43-47.

33 Topcuoglu MA, Aydin H, Saka E: Occipital cortex activation studied with simultaneous recordings of functional transcranial Doppler ultrasound (fTCD) and visual evoked potential (VEP) in cognitively normal human subjects: effect of healthy aging. Neurosci Lett 2009;452:17-22.

-34 Zaletel M, Strucl M, Pretnar-Oblak J, Zvan B: Age-related changes in the relationship between visual evoked potentials and visually evoked cerebral blood flow velocity response. Funct Neurol 2005;20:115-120.

>35 Helzner EP, Luchsinger JA, Scarmeas N, Cosentino S, Brickman AM, Glymour MM, Stern Y: Contribution of vascular risk factors to the progression in Alzheimer disease. Arch Neurol 2009;66:343-348.

-36 Kimberly WT, Gilson A, Rost NS, Rosand J, Viswanathan A, Smith EE, Greenberg SM: Silent ischemic infarcts are associated with hemorrhage burden in cerebral amyloid angiopathy. Neurology 2009;72:1230-1235. 